\title{
Experiment in 3D Vision based Hovering Control of an Autonomous Helicopter*
}

\author{
Zhenyu YU**, Liang TANG** and Kenzo NONAMI*** \\ ${ }^{* *}$ Graduate School of Engineering, Chiba University \\ 1-33 Yayoi-cho, Inage-ku, Chiba, Japan \\ E-mail:yzy@graduate.chiba-u.jp \\ *** Depatment of Electronics and Mechanical Engineering,Chiba University \\ 1-33 Yayoi-cho, Inage-ku, Chiba, Japan
}

\begin{abstract}
This paper describes our experimental study in hovering control of an unmanned helicopter with a 3D vision system instead of GPS(Global Position System). The aim of this study is to investigate methods to enable the normal operation of an autonomous helicopter when GPS does not function properly as is often experienced in cluttered environment. A 3D vision system together with a convex colored landmark is used for estimating the position of the vehicle. The estimated position is then fused with the acceleration from IMU (Inertial Measurement Unit) by Kalman filter for velocity estimation. The sensing strategy is validated by field experiments. The helicopter is stably controlled to hover within a 0.4 meter radius circle around the reference point. The hovering performance is equivalent to that when GPS is used. The good hovering performance indicates a possible application of vision system as an alternative of GPS in the case when GPS has trouble to provide reliable measurement.
\end{abstract}

Key words: Vision Based Hovering Control, Unmanned Helicopter, Convex Colored Landmark, Sensor Fusion

\section{Introduction}

Unmanned Aerial Vehicles (UAVs) are promising utilities for dirty, dull, and dangerous tasks. Especially, unmanned helicopters which can hover, take off and land vertically, see applications in space constrained or maneuver limited situations such as disaster survey, search and rescue, communication relay, power transmission line inspection and so on ${ }^{(1)}$. More and more civil applications are expected to emerge in the near future as the technology advances in this area ${ }^{(2)}$. Attitude and position information are important for UAV control decision. The attitude measurement normally comes from IMU, and position is usually measured with a GPS in either standalone mode or differential mode ${ }^{(3),(4),(5)}$.

The availability of GPS has made the position and velocity measurement an easy task. GPS works relying on the communication with satellites. For successful operation, it requires a clear view of the sky with at least 4 satellites in sight. It is not favorable to apply GPS in situations where the requirement may be violated. Flying around buildings or among mountains is the case that GPS more likely fails to provide continuously stable measurement. The discontinuity, however, may lead to fatal problem in vehicle's stability. Using vision system for position sensing is one method which has drawn much attention in recent years. Existing methods include estimating the position by using natural features ${ }^{(6),(7)}$ or artificial patterns ${ }^{(8),(9)}$. It is advantageous to use natural features since it puts fewer limits on the application environment. However the inherent computational complexity makes it 
negative for real-time control purpose. Amidi et. al. have developed a visual odometer for position sensing with a pair of video cameras and gyroscopes ${ }^{(6)}$. The system makes use of natural features for matching and finding the relative displacement between image frames, and it is built with a dedicated hardware to be able to measure at $60 \mathrm{~Hz}$. Shakernia et. al. present a monocular vision system with a carefully designed pattern pad for landing an unmanned helicopter ${ }^{(8)}$. A monocular vision system based on Moiré patterns has been used for controlling a quadrotor indoor vehicle ${ }^{(9)}$. The system estimates the position by utilizing geometry and four single-point discrete Fourier transforms on the morié patterns.

The approach presented in this paper is based on a COTS (Commercial Off The Shelf) binocular stereo vision system. A simple convex-shaped color blob is used as position reference. The HSI (Hue, Saturation, and Intensity) color model is adopted for color analysis. The algorithm performs profile analysis on the hue component to find the colored landmark so as to determine the helicopter's position. The algorithm is simple and computational efficient for real time application, and it is successfully applied in hovering control of an autonomous helicopter in the absence of GPS.

The paper is organized as follows. Section 2 introduces the helicopter platform and hardware components used in the study. Section 3 describes how to determine position from a convex-shaped color landmark with a stereo camera. Section 4 shows velocity estimation with vision and IMU. Section 5 briefs the control system and the experimental results. Section 6 concludes the study and discusses further direction.

\section{The research helicopter}

The helicopter platform is based on a 40cc gasoline engine powered model helicopter. The specification is shown in Table 1. A fully instrumented helicopter is shown in Fig.1.

Table 1 Specification of the research helicopter

\begin{tabular}{c|c}
\hline \hline Item & Description \\
\hline Fuselage length & $1467 \mathrm{~mm}$ \\
Fuselage width & $245 \mathrm{~mm}$ \\
Main rotor span & $1790 \mathrm{~mm}$ \\
Tail rotor span & $273 \mathrm{~mm}$ \\
Net weight & $9 \mathrm{kgf}$ \\
Max. Payload & $8 \mathrm{kgf}$ \\
\hline \hline
\end{tabular}

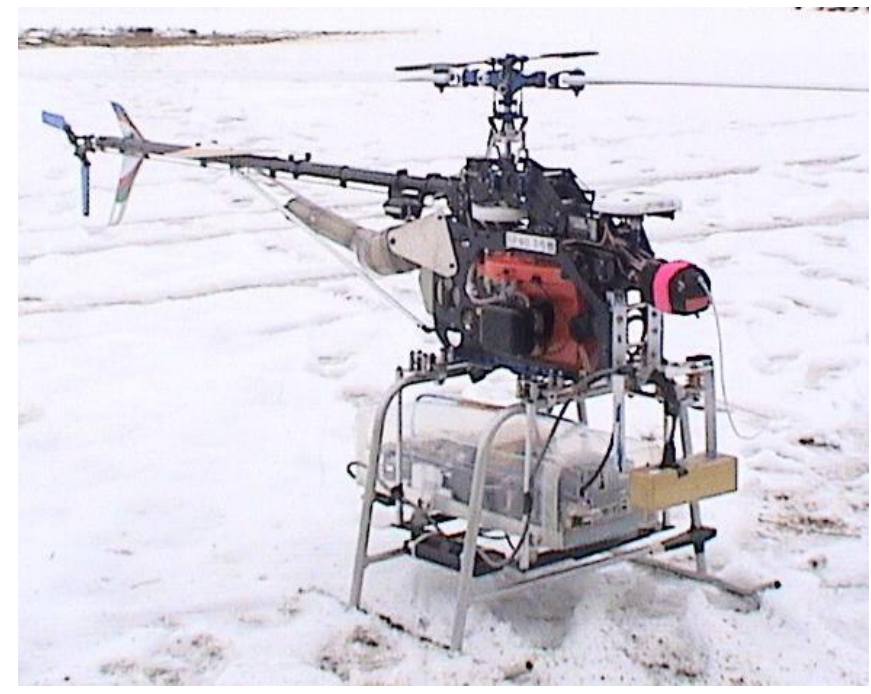

Fig.1 The helicopter with experimental equipment 
Remote control of the helicopter is realized by a set of servomotors, radio receiver and transmitter. Flying command is sent to the servomotors through the pair of radio transmitter and receiver. The servomotors then actuate the throttle, main collective pitch, cyclic pitch and tail collective pitch accordingly so as to control the motion of the helicopter. The throttle controls engine power; main collective pitch controls the main rotor thrust; cyclic pitch controls the direction of main rotor thrust thus the roll, pitch, forward-backward and left-right motion; the tail collective pitch controls the yaw motion by changing the tail thrust. In manual control mode, 4 channels are used. They are throttle/pitch, aileron (controls roll and left-right motion), elevator (controls pitch and forward-backward motion) and rudder (controls yaw motion). The throttle and main collective pitch is controlled by one channel. The individual control is realized by preprogrammed static mappings between stick movement /collective pitch and stick movement / throttle respectively.

The autonomous control mode is implemented by taking advantage of the existing remote control infrastructure. The radio control system is still used for sending control command. The control authority is taken by the flight computer, which makes control decision based on the information from sensors.

The sensors used for implementing autonomous flight include an AHRS (Attitude Heading Reference System), and a 3D vision system. The total weight of the onboard system is $4.5 \mathrm{~kg}$. The specifications of the sensors are shown in Table 2.

Table 2 Specifications of the sensors in use

\begin{tabular}{c|ll}
\hline \hline Sensor Name & \multicolumn{1}{c}{ Specification } \\
\hline & $\bullet$ & Size: $7.62 \mathrm{~cm} \times 9.53 \mathrm{~cm} \times 10.42 \mathrm{~cm}$ \\
AHRS & $\bullet$ & Weight: $770 \mathrm{~g}$ \\
(Crossbow AHRS400) & $\bullet$ & Interface: $\mathrm{RS}-232$ \\
& $\bullet$ & Update rate: $>60 \mathrm{~Hz}$ \\
& $\bullet$ & Power consumption: $<4 \mathrm{~W}$ \\
\hline & $\bullet$ & Baseline: $12 \mathrm{~cm}$ \\
& $\bullet$ & Resolution: $1024 \times 768$ \\
Stereo vision system & $\bullet$ & Max. frame rate: $15 \mathrm{fps}$ \\
(Point Grey Bumblebee) & $\bullet$ & Horizontal field of view: $50^{\circ}$ \\
& $\bullet$ & Interface: IEEE 1394 \\
& $\bullet$ & Weight: $375 \mathrm{~g}$ \\
& $\bullet$ & Power consumption: $2.1 \mathrm{~W}$ \\
\hline \hline
\end{tabular}

\section{Localization with a colored landmark}

\subsection{Overview}

Vision system captures local information in the view. Unlike GPS which provides measurement under a global coordinate system i.e. WGS-84, vision system can only provide measurement in a local coordinate system. The position can be determined with the help of dynamic ${ }^{(6),(7)}$ or fixed reference ${ }^{(8),(9)}$. In this research, a fixed reference is used. It is a convex colored landmark. The algorithm for positioning includes color extraction, landmark detection, and coordinate transformation.

Four coordinate systems are used in describing the algorithm. They are world frame $\mathrm{o}_{\mathrm{L}}-\mathrm{x}_{\mathrm{L}} \mathrm{y}_{\mathrm{L}} \mathrm{Z}_{\mathrm{L}}$, helicopter body frame $\mathrm{o}_{\mathrm{H}}-\mathrm{x}_{\mathrm{H}} \mathrm{y}_{\mathrm{H}} \mathrm{Z}_{\mathrm{H}}$, camera frame $\mathrm{o}_{\mathrm{C}}-\mathrm{x}_{\mathrm{C}} \mathrm{y}_{\mathrm{C}} \mathrm{Z}_{\mathrm{C}}$ and image frame $\mathrm{o}_{\mathrm{I}}-\mathrm{x}_{\mathrm{I}} \mathrm{y}_{\mathrm{I}}$. The origin of the world frame locates at the C.G. (Center of Gravity) of the landmark and it is used to express the helicopter's inertial position. The helicopter body frame fixes at the C.G. of the helicopter and the camera frame is attached to the camera, which has a rigid relationship with the helicopter body frame. The image frame is used to express pixels in the image plane. The definitions of the frames are illustrated in Fig. 2. The axes which are 
not shown in Fig. 2 are determined by right hand rule.

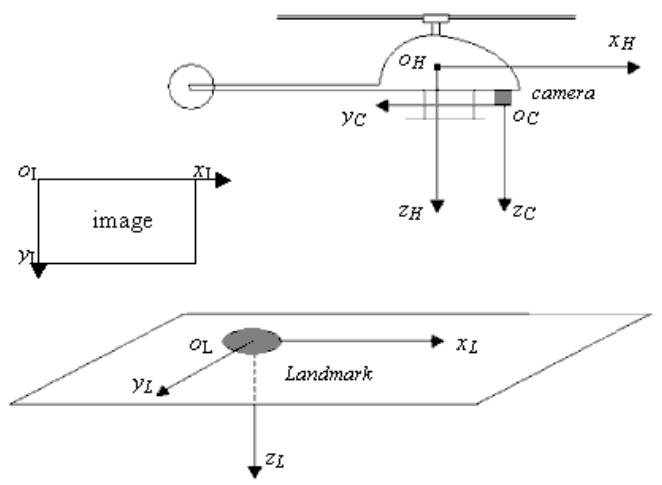

Fig.2 Definitions of the coordinate systems

\subsection{Convex landmark}

A convex landmark has the property that the C.G. (Center of Gravity) point lies in its interior. And its profile features bell-shape like curve in any orthogonal projecting axes. With these properties, the landmark can be detected efficiently in the sense of computational complexity by analyzing its profiles. The profile expresses the image's distribution along axis of interest. The profiles along the image's coordinate axes are often used.

Let $I(i, j), 0 \leqslant i<M, 0 \leqslant j<N$, where $M$ is the image's row number, $N$ is the image's column number. The image profile on horizontal axis $H(i)$ and vertical axis $V(j)$ are expressed in Eq.(1) and Eq.(2) respectively.

$$
\begin{aligned}
& H(i)=\sum_{j=0}^{M-1} I(i, j), 0 \leq i<N \\
& V(j)=\sum_{i=0}^{N-1} I(i, j), 0 \leq j<M
\end{aligned}
$$

To aid the shape analysis, the horizontal and vertical profiles are divided into intervals alone their profiling axes. The intervals whose values are greater than zero are defined as span. The span of horizontal profile and vertical profile are defined in Eq. (3).

$$
\begin{aligned}
& S_{H}:\{(a, b) \mid a<b, H(a)=0, H(b)=0, H(i)>0 \text { for } i \in(a, b)\} \\
& S_{V}:\{(a, b) \mid a<b, V(a)=0, V(b)=0, V(i)>0 \text { for } i \in(a, b)\}
\end{aligned}
$$

The span width associated with each span element is defined as Eq.(4).

$$
W=b-a \text {, where }(a, b) \in S_{H} \text { or }(a, b) \in S_{V}
$$

Examples of convex and non-convex shapes are shown in Fig.3(a), and the definition of profile span is illustrated in Fig.3(b)

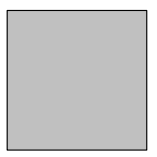

convex

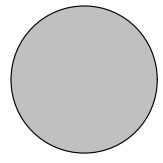

convex

(a)

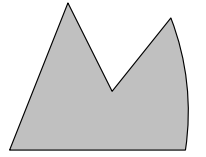

non-convex

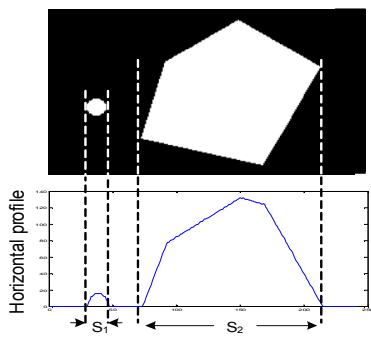

(b)

Fig.3 (a) Convex and non-convex shapes; (b) Profile span definition. The profile span contains intervals $S_{1}$ and $S_{2}$ 


\subsection{Color recognition}

The processing stage first transforms the RGB image into its HSI representation through Eq. (5) to Eq. (7), and then apply threshold on the hue channel to separate the target color out of the background. The result is a binary image with the white indicates the recognized color area.

$$
\begin{aligned}
& I=\frac{1}{3}(R+G+B) \\
& S=1-\frac{3}{(R+G+B)} \min (R, G, B) \\
& H=\cos ^{-1}\left[\frac{\frac{1}{2}[(R-G)+(R-B)]}{\sqrt{(R-G)^{2}+(R-B) \cdot(G-B)}}\right] \\
& H=2 \pi-H \text { if } B>G
\end{aligned}
$$

$H$ is normalized by dividing its value by $2 \pi$.

In HSI color model, the color information is encoded by hue and saturation. Hue describes a pure color, whereas saturation gives a measure of the degree to which a pure color is diluted by white light. The HSI model decouples the color information from the intensity. The hue value indicates the dominant wavelength of light waves reflected by the object and it is used to recognize the target color in the study.

The unwanted colors are filtered off by applying threshold on the image's hue component. The threshold $T$ is statistically determined by analyzing the hue value of images. Then it is converted to a binary image $B(i, j)$ based on the following rule shown in Eq. (8).

$$
B(i, j)=\left\{\begin{array}{l}
1, \text { when } \mid \text { Hue }[I(i, j)]-T \mid<\delta \\
0, \text { otherwise }
\end{array}\right.
$$

where $\delta$ is a small positive number to accept some small variations.

\subsection{Landmark detection}

The landmark detection is primarily based on the color information which is properly chosen not to be homogeneous with the background. Additional clues that help the detection are from the profile analysis. When a convex landmark is specified, its profiles can be used as templates for shape matching. The profile span width should be compatible with the landmark's geometry. This fact is used as evidence for landmark detection.

The algorithm works on the binary image $B(i, j)$ resulted from color recognition step. The landmark decision involves the following procedure:

a) Perform profile analysis on image $B(i, j)$ to get its horizontal profile $H(i)$ and vertical profile $V(j)$.

b) Remove spike noise with small magnitude by threshold based on Eqs.(9) and (10).

$$
\begin{aligned}
& H(i)=\left\{\begin{array}{c}
H(i), \text { if } H(i)>M I N \\
0, \text { else }
\end{array}\right. \\
& V(j)=\left\{\begin{array}{c}
V(j), \text { if } V(j)>M I N \\
0, \text { else }
\end{array}\right.
\end{aligned}
$$

where $M I N$ is the minimum valid profile strength.

c) Filter out spike noise with large magnitude by using the profile span condition. Only the intervals of the profiles with span width in between the valid range of the landmark are kept for further processing. The valid intervals are stored as $X$ and $Y$ 
respectively.

$$
\begin{aligned}
& X:\left\{\left(a_{i}, b_{i}\right) \mid\left(a_{i}, b_{i}\right) \in S_{H}, i=1,2,3, \cdots, p\right\} \\
& Y:\left\{\left(c_{i}, d_{i}\right) \mid\left(c_{i}, d_{i}\right) \in S_{V}, i=1,2,3, \cdots, q\right\}
\end{aligned}
$$

where $p$ and $q$ are the horizontal and vertical interval numbers respectively.

Each candidate is specified by its coordinates $(x, y)$ in the image, where $x \in X, y \in Y$.

d) Check each candidate's area and remove those whose areas are beyond the valid range determined statistically from experimental images. The area of the candidate is calculated by Eq. (12).

$$
A=\sum_{i=a}^{b} \sum_{j=c}^{d} B(i, j)
$$

where $a, b, c, d$ are specified by the candidate's coordinate $(x, y)$.

e) If no valid candidate is left, the process repeats on the next image. If more than one valid candidate is found, other features of the landmark can be used for recognition. However, in this research, this case is treated as invalid, since the assumption on landmark's color setting and the color recognition process make it a rare case. The C.G. point of the valid landmark is calculated by Eq.(13) and used as index to find its $3 \mathrm{D}$ position.

$$
\begin{array}{r}
\bar{x}=\frac{\sum_{i=a}^{b} \sum_{j=c}^{d} i \times B(i, j)}{\sum_{i=a}^{b} \sum_{j=c}^{d} B(i, j)} \\
\bar{y}=\frac{\sum_{i=a}^{b} \sum_{j=c}^{d} j \times B(i, j)}{\sum_{i=a}^{b} \sum_{j=c}^{d} B(i, j)}
\end{array}
$$

Based on the algorithm, several tests are performed to check the performance. In the processing of total 900 images under different light and background conditions, the success rate is $90 \%$. In Fig. 4, some recoginition results are presented.

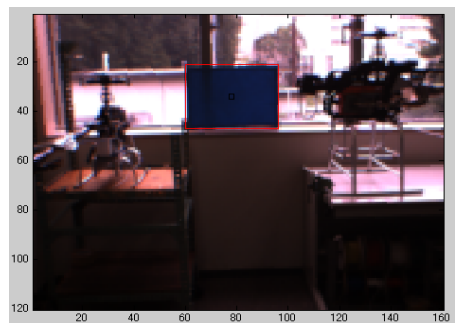

(a) Indoor test with dark light

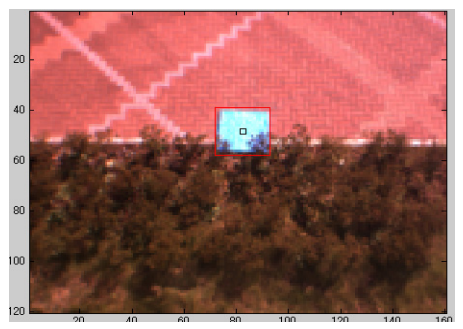

(c) Outdoor test with mixed background

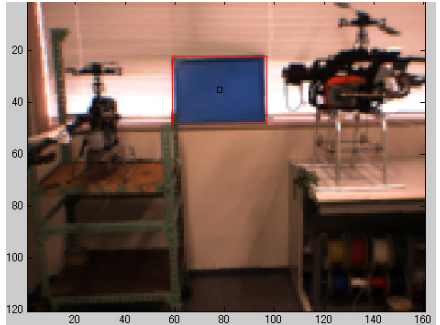

(b) Indoor test with bright light

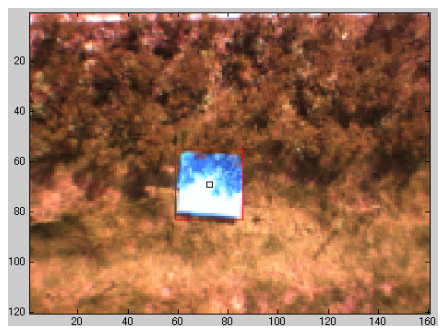

(d) Outdoor test in grass

Fig. 4 Landmark detection result. The red rectangle indicates successful detection. 


\subsection{Coordinate transform with delay compensation}

Since the reference point is determined in the image, the 3D position can be retrieved from the 3D data generated by stereo vision system with the reference point as index. The position data is with respect to the camera frame and should be transformed to world frame before feeding to the controller. This transformation can be done with the attitude data measured by AHRS.

However, the AHRS and the vision system run separately. The data coming from the two systems are not in synchronization. In order to use the right attitude data for transformation, we need find the phase difference between the position measured by vision and the attitude measured by AHRS. We have designed an identification test for the purpose. The vision system and the AHRS are mounted on an aluminum plate. The plate is placed on a $1.8 \mathrm{~m}$ high table with a landmark underneath. Figure 5 illustrates the test configuration. The plate is shaken in roll direction and the data are logged.

By using the coordinates of the reference point determined from vision, the roll angle can be calculated from vision measurement by Eq.(14). As the same time the roll angle can be measured by AHRS. Let $\phi^{\prime}$ and $\phi$ denote the calculated roll angle by vision and that measured by AHRS respectively, the phase delay of vision data with respect to the AHRS data can be found by comparing the two roll measurements.

$$
\phi^{\prime}=\arctan \left(\frac{x}{z}\right) \times \frac{180}{\pi}
$$

where $(x, y, z)$ is the landmark's coordinate in the camera's frame.

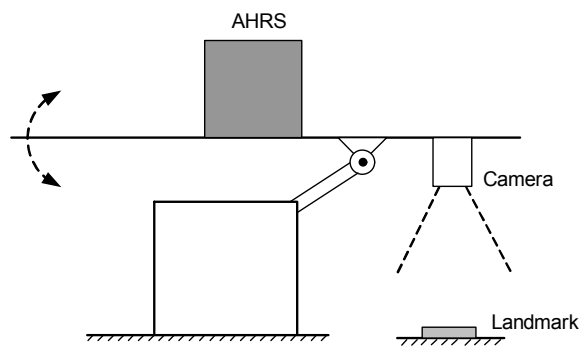

Fig.5 Test setup for delay measurement between AHRS and camera

The phase delay $T_{d}$ between the two roll measurements is identified by cross correlation analysis. The cross correlation $R$ is computed by Eq. (15).

$$
R(\tau)=\int_{0}^{T} \phi^{\prime}(t) \phi(t+\tau) d t
$$

where $T$ is the time length of the data used for analysis. The time delay can be determined by Eq. (16).

$$
T_{d}=\arg \max _{\tau} R(\tau)
$$

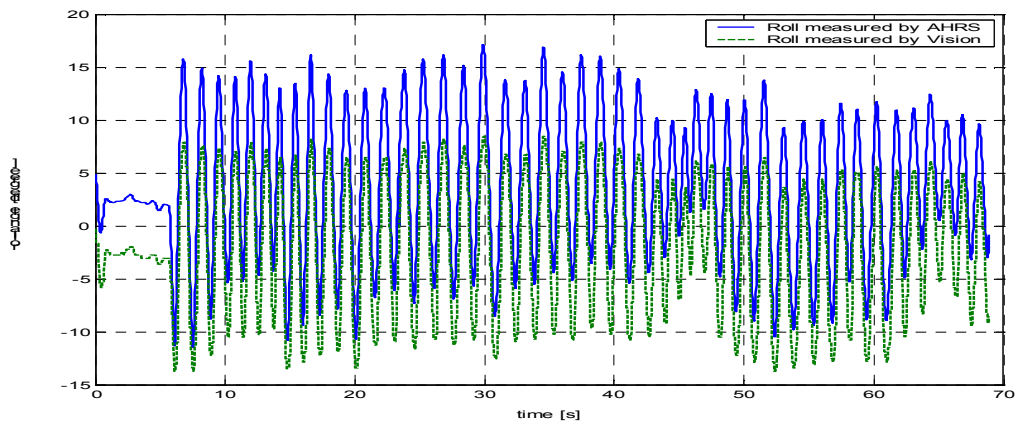

Fig.6 Roll measured by AHRS and vision 
In Fig. 6 we show the roll measured by vision system and AHRS respectively. The blue line shows the measured roll angle by AHRS and the green line indicates the calculated roll angle by using position measured by vision system. It is observed that the two measurements match in the sense of dynamic change. However, there is an offset between them. It is because that the "zeros" of two sensors are not in alignment. We can make them agree by putting the landmark with zero offset from the camera's optical axis while keeping the plate where the roll angle measured by AHRS is zero. This calibration process will ensure zero offset between the two measurements, but it is not necessary in our case since the purpose is to find the delay between the two measurements and the systematic bias error does not affect the result.

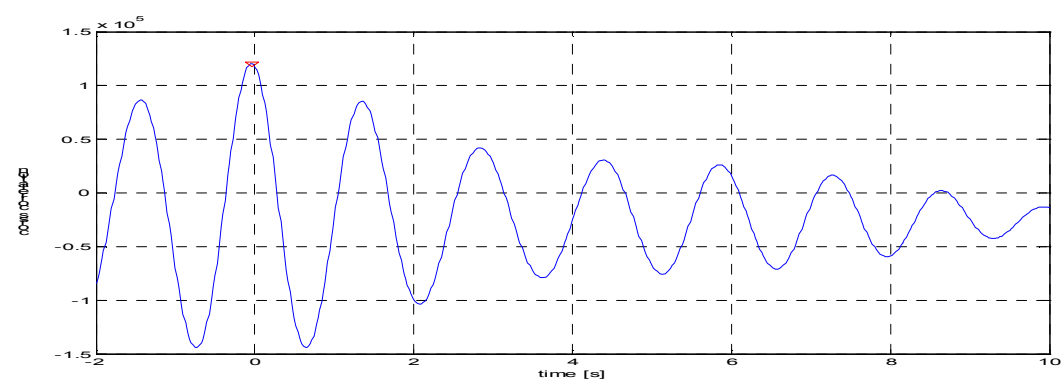

Fig. 7 Cross correlation result between roll measurements

The time delay is identified from the cross correlation result as shown in Fig. 7. The red triangle mark in the figure indicates that the vision measurement is $40 \mathrm{~ms}$ later than the IMU data. It means that the corresponding attitude data of current vision measurement are the ones $T_{d}$ seconds ago. The right attitude data for coordinate transform can be obained by artifically delaying the IMU measurement. The concept is illustrated in Fig. 8.

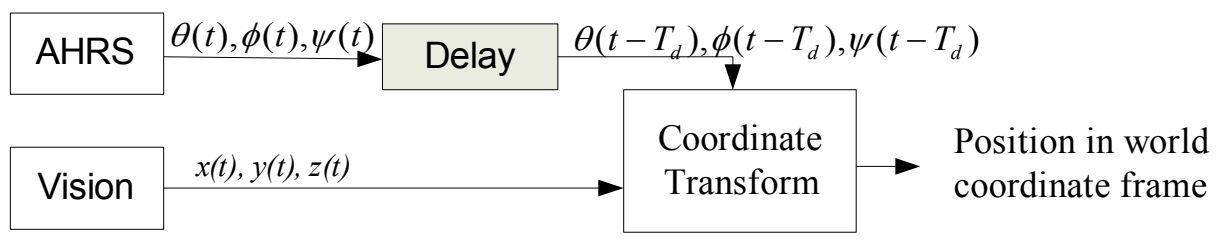

Fig. 8 Illustration of delay compensation

\section{Velocity estimation}

In the case of GPS based control, both position and velocity are used as feedback measurements. Since the controller has been verified through field experiements in previous study $^{(3)}$, we plan to use the existing controller to evaluate the vision based sensing stragety. As discussed in the previous section, the vision system gives the position estimate of the helicopter with respect to the landmark. To be able to use the existing controller, we have to provide the velocity estimation.

The velocity is estimated with Kalman filter by fusing the position data and the acceleration measurement from IMU. In the Kalman filter design model, the acceleration from IMU is treated as process input and the position from camera as measurement. The acceleration noise is modeled as slow changing bias $\xi$ plus white noise $w_{l}$. The bias $\xi$ is described by a first order Markov dynamic system driven by white noise $w_{2}$, of which the time constant is $T_{b}$. The measurement noise is modeled as white noise $w_{3}$. The model used for estimating the velocity in $x$ axis is shown in Eq. (17). The model for $y$ axis is in the same form and is not shown again.

In Eq.(17), $x^{w}$ denotes the position and $a_{x}^{w}$ represents the acceleration in world 
frame.

$$
\left\{\begin{array}{l}
{\left[\begin{array}{l}
\dot{x}^{w} \\
\ddot{x}^{w} \\
\dot{\xi}
\end{array}\right]=\left[\begin{array}{ccc}
0 & 1 & 0 \\
0 & 0 & -1 \\
0 & 0 & -\frac{1}{T_{b}}
\end{array}\right]\left[\begin{array}{c}
x^{w} \\
\dot{x}^{w} \\
\xi
\end{array}\right]+\left[\begin{array}{l}
0 \\
1 \\
0
\end{array}\right] a_{x}^{w}+\left[\begin{array}{cc}
0 & 0 \\
1 & 0 \\
0 & \frac{1}{T_{b}}
\end{array}\right]\left[\begin{array}{l}
w_{1} \\
w_{2}
\end{array}\right]} \\
y=x^{w}+w_{3}
\end{array}\right.
$$

Based on the model, a steady state Kalman filter is designed. To evaluate its performance, we compare it with the velocity measured by the GPS which was used in our previous hovering control. The velocity accuracy of the GPS is $0.043 \mathrm{~m} / \mathrm{s}$ (RMS). A plot of static test of the GPS is shown in Fig. 9.

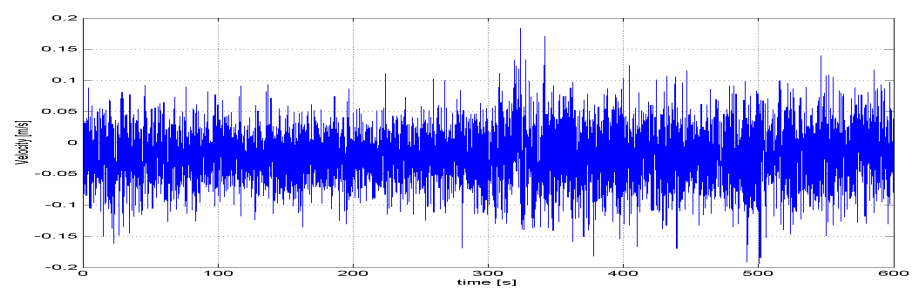

Fig. 9 Time history of the measured velocity by GPS at still condition

In the fact that good hovering performance was achieved based on the same GPS, it is reasonable to expect similar result could be obtained if vision based measurements are of the same grade in quality as those of GPS. The comparision result is shown in Fig. 10.

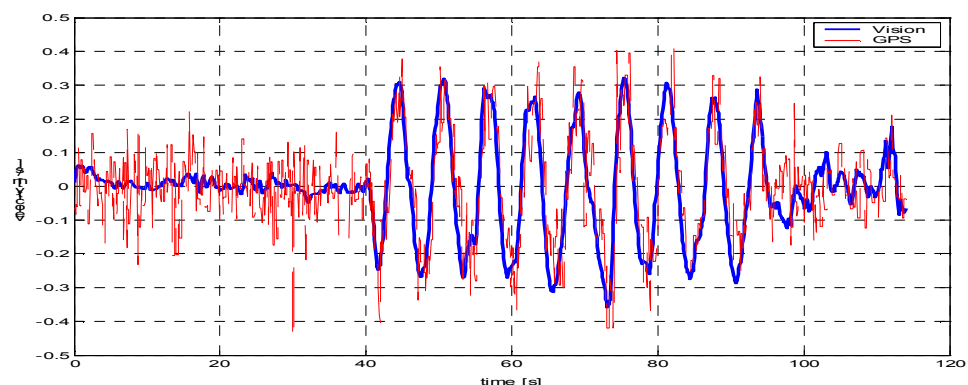

Fig. 10 Velocity measured by GPS and that estimated by using vision and IMU

During the first 40 seconds, it was stationary test. The vision based estimation is shown with less variance than GPS. The dynamic test from 40s to $96 \mathrm{~s}$ shows well agreement between the two. After that it was brought to rest again. Compared to GPS, the vision based estimation returned to zero slower. This is due to the fact that after the stop of dynamic test the equipment still experienced some small movement. The measured acceleration was negative as shown in the acceleration plot in Fig.11. This contributed to the observed result.

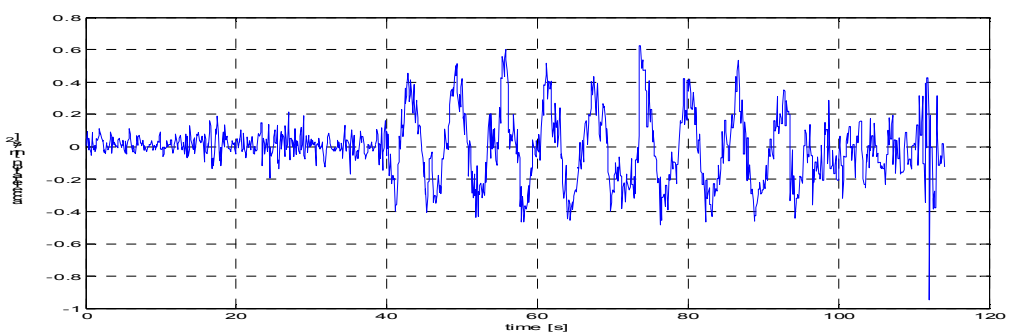

Fig.11 Acceleration during the test 
The result demonstrates that the estimated velocity is comparable to GPS measurement in hovering case. It indicates the feasibility to replace GPS velocity with the estimated one from vision and IMU. The approach is further verified by our field experiement which is explained in next section.

\section{Hovering control}

The translational motion of a helicopter is induced by the change of thrust vector of the main rotor. As a result, attitude control is crucial for translational motion control. The previous study at the laboratory to which the authors belong has identified attitude model and translational motion model for a small helicopter near hovering condition $^{(3),(4)}$. The models are successfully applied for designing hovering controllers. It is observed that the bandwidth of attitude dynamics is higher than that of linear motion dynamics. By time scale separation principle, the controller is designed with an inner attitude control loop and an outer translational motion control loop. Both attitude controller and translational motion controller are synthesized by using linear quadratic programming method. In Fig.12 we show the block diagram of the controller configuration.

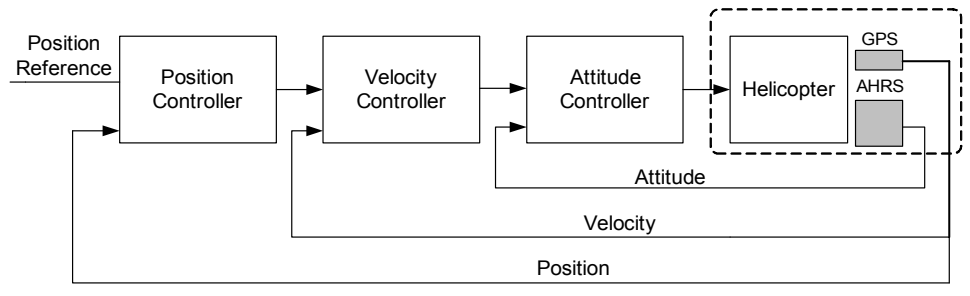

Fig. 12 The applied controller configuration ${ }^{(3)}$

In the previous study ${ }^{(3),(4)}$, the attitude is sensed by the IMU and the position and velocity are measured by GPS. The vision based hovering control is implemented by the same control stragety whereas the role of GPS used before is replaced by the vision system for position and velocity measurements. The reason that we use the same control stragety as previous study is because the vision system achieves comparable performance as GPS in measuring position and velocity in the hovering case. The discussion on the detailed controller design is well described in references ${ }^{(3),(4)}$. The block diagram of the vision based hovering control is shown in Fig.13.

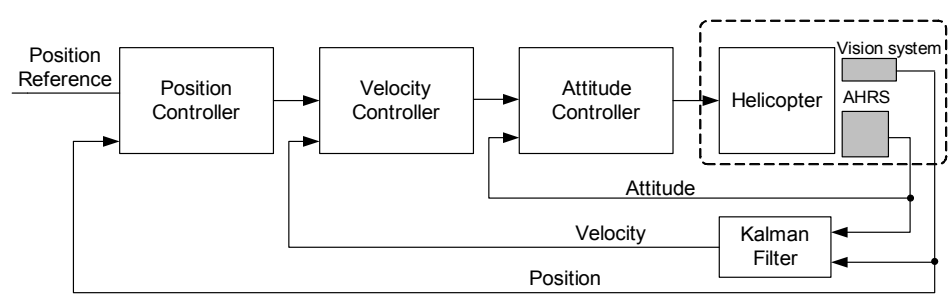

Fig.13 Configuration of the vision based controller

\section{Experiment}

Field flight test was carried out to study the performance of the proposed approach. The reference landmark used is a square wooden board with lateral length of 0.7 meter. The board is covered by a blue sheet which is the target for color detection. The helicopter is to be controlled to hover above the landmark autonomously. The flying height is about $5 \mathrm{~m}$ above the ground. The image processing is done on Ampro 800 single board computer which is configured with a $1.4 \mathrm{GHz}$ PentiumM CPU and $1 \mathrm{~GB}$ memory. The image resolution is set to be 160 by 120 pixels. The image processing takes $65 \mathrm{~ms}$, and this gives an update rate of about $15 \mathrm{~Hz}$. Figure 14 shows a scene of the experiment at the time when the helicopter hovers stably by the vision based controller.

Before the autonomous control starts, the helicopter is controlled to fly over the 
landmark manually. When the landmark is in the view of the camera and is successfully detected, the control authority is switched to autonomous controller. From then on the controller makes the control decision based on the information provided by the attitude sensor and the vision system. The experimental result is shown in Fig. 15. To evaluate the performance, the hovering result is compared to that by using GPS instead of vision system as shown in Fig. 16. Both experiments were performed in no wind conditions but in different days.

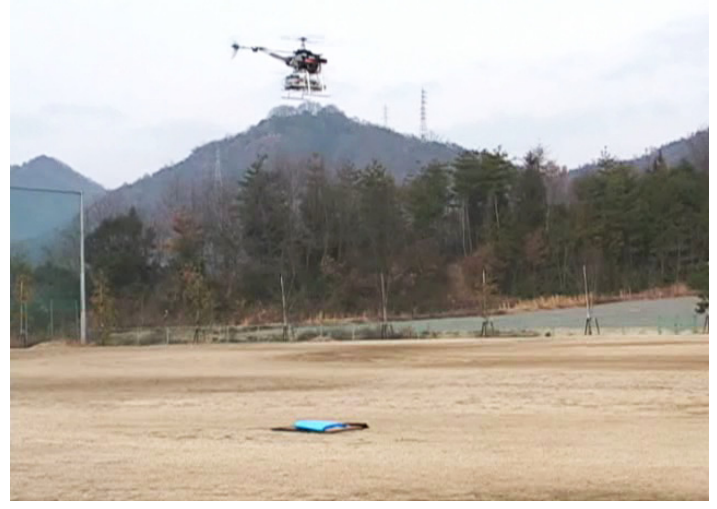

Fig.14 Autonomous hovering above a landmark

It is observed that the trajectory of vision based hovering lies in the circle around the landmark with radius of $0.4 \mathrm{~m}$. In the GPS based hovering case, the trajectory is within the circle with radius of $0.3 \mathrm{~m}$. The vision based hovering performance is comparable to the GPS based hovering performance. From the comparison, it is also observed that the GPS based hovering is more stable. The cause under consideration is the fact that the onboard system for vision based control is heavier than that for GPS based control and the imperfect mounting leads to longitudinal shift of the helicopter's C.G. point which gives rise to the result observed. The difference in configuration of onboard system is that in the GPS based control case, the camera was not attached which is $375 \mathrm{~g}$. The longitudinal variation could be reduced if the mounting effect on the C.G. change was mitigated.

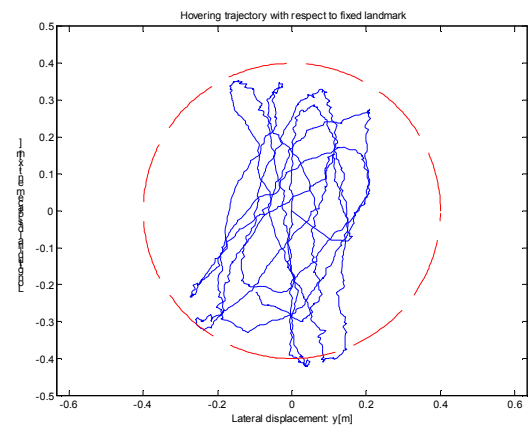

Fig.15 Helicopter's trajectory in vision based hovering case

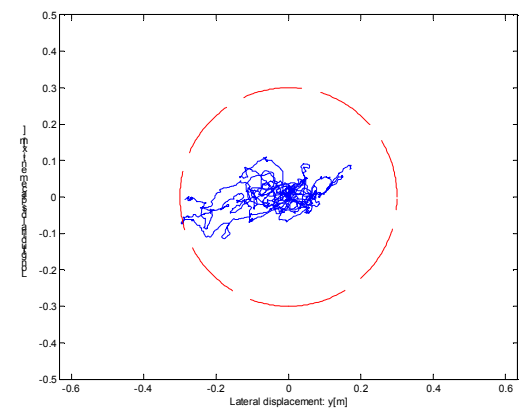

Fig.16 Helicopter's trajectory in GPS based hovering case

\section{Conclusion}

In this study, a 3D vision based relative positioning with a color landmark is proposed. The measurement error is bounded by the dimension of the landmark when the landmark is detected. The approach has been successfully applied for hovering control of an unmanned helicopter. The experimental result demonstrates good hovering performance. The hovering trajectory lies in the circle with radius of $0.4 \mathrm{~m}$. The result of the study suggests the 
possibility of using vision system for hovering control where GPS is not available. Some possible applications requiring hovering capability without GPS may include communication relay in urban area, aerial photography at fixed point, indoor application and so on. However, the requirement of landmark does put constraint on the applications. Future work will emphasize on extracting image pattern on the fly and position by tracking the extracted pattern.

\section{References}

(1) M. Selier and G. Voorsluijs, Future Surveillance using autonomous unmanned helicopters. AIAA/ICAS International Air and Space Symposium and Exposition: The next 100 years, (2003).

(2) K. Nonami, Prospect and Recent Research \& Development for Civil Use Autonomous Unmanned Aircraft as UAV and MAV, Journal of System Design and Dynamics, Vol.1, No.2, (2007), pp.120-128.

(3) K. Hazawa, J. Shin, D. Fujiwara, K. Igarashi, D. Fernando, and K. Nonami, Autonomous Flight Control of Small Hobby-Class Unmanned Helicopter, Report2: Modeling based on Experimental Identification and Autonomous Flight Control Experiments,Journal of Robotics and Mechatronics, Vol.15, No.5,(2003), pp.546-554.

(4) J. Shin,D. Fujiwara, K. Hazawa and K. Nonami, Model Based Optimal Attitude and Positioning Control of Small-Scale Unmanned Helicopter, Trans. of the Japan Society of Mechanical Engineers, Vol.70, No.697, C, (2004), pp.2631-2637

(5) K. Sprague, V. Gavrilets, D. Dugail, B. Mettler, I. Martinos, and E. Feron, Design and applications of an avionics system for a miniature acrobatic helicopter. In Digital Avionics Systems Conference, (2001).

(6) O. Amidi, T. Kanade and K. Fujita, A visual odometer for autonomous helicopter flight. Robotics and Autonomous Systems, Vol.28, No.2, (1999), pp.185-193.

(7) T. Kanade, O. Amidi and Q. Ke, Real-time and 3D vision for autonomous Small and Mirco Air vehicles. In 43rd IEEE conference on Decision and Control, (2004).

(8) O. Shakernia, Y. Ma, J. Koo and S.S. Sastry, Landing an Unmanned Aerial Vehicle: Vision Based Motion Estimation and Nonlinear Control. Asian Journal of Control, Vol.1, No.3, (1999), pp.128-145.

(9) G.P. Tournier, M. Valenti, J.P. How and E. Feron, Estimation and control of a quadrotor vehicle using monocular vision and Moiré Patterns. In AIAA Guidance, Navigation and Control Conference and Exhibit, 21-24 August 2006, Keystone, Colorado, (2006). 